\title{
Magnetic structures and their determination using group theory
}

\author{
A. Wills \\ Institut Laue-Langevin, BP. 156x, 38042 Grenoble cedex, France
}

\section{Introduction}

The determination of magnetic structures is a special area of condensed matter research. While being fundamental to the understanding of electronic structures and properties, it remains a subject that is treated with difficulty and is full of incorrect solutions. This article is based around two goals:

- The explanation of the different possible types of magnetic structure.

- The demonstration of how symmetry leads to their proper description, and can aid their solution.

In content, the first part of this article is based on the practicalities of what an experimenter should know in order to understand and describe a magnetic structure. In the second part, symmetry arguments will be shown to reduce the otherwise arduous task of determining a magnetic structure, to the investigation of a handful of possible structures.

\section{Basic crystallography}

\subsection{Nuclear crystal structures}

A nuclear crystal structure can be described in terms of lattice translations of a unit cell. If the unit cell contains only one atom it is said to be a primitive cell; if it contains several atoms it is said to be a non-primitive lattice. The atomic positions of an arbitrary atom in the $l$ th unit cell is given by

$$
\mathbf{R}_{\mathrm{t} \mathbf{j}}=\mathbf{t}+\mathbf{r}_{\mathbf{j}}
$$

where

$$
\mathbf{t}=n_{1} \mathbf{a}+n_{2} \mathbf{b}+n_{3} \mathbf{c}
$$

and

$$
\mathbf{r}_{j}=x \mathbf{a}+y \mathbf{b}+z \mathbf{c}
$$

Here $a, b, c$ are unit vectors of the nuclear cell defined according to the International Tables; $n_{1}, n_{2}, n_{3}$ are integers and $x, y, z$ have values that are less than unity. 


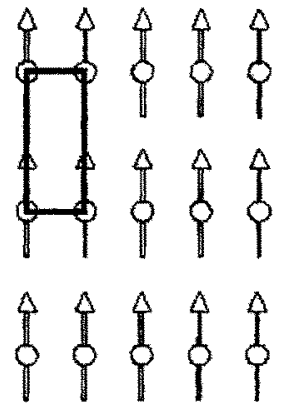

A) ferromagnetic

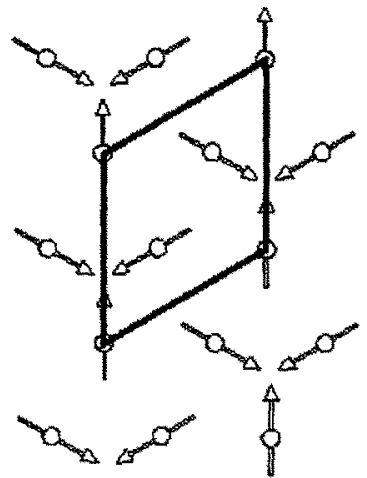

d) triangular

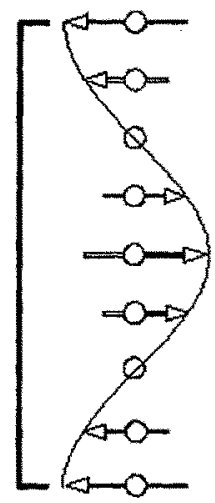

h) sine or cosine

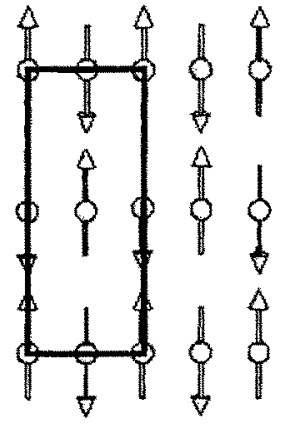

b) antiferromagnetic


e) canted
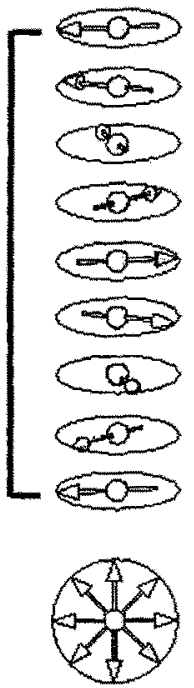

i) circular helix

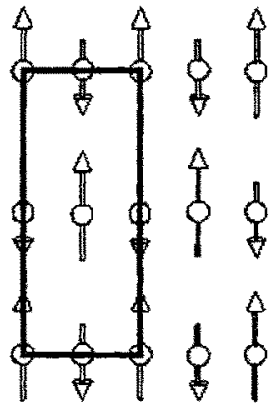

c) ferrimagnetic


fumbrella
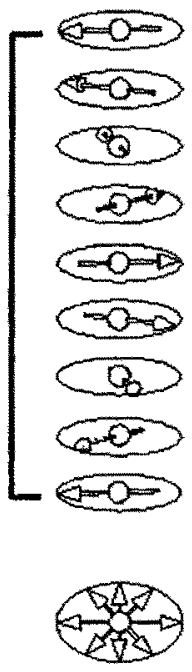

j) elliptical helix

Figure 1: Some different types of magnetic structures 


\subsection{Reciprocal lattice}

In crystallography a useful and much used construction is the 'reciprocal lattice'- this can be defined as:

$$
\begin{aligned}
\mathbf{a}^{*} & =\frac{2 \pi}{v_{0}} \mathbf{b} \wedge \mathbf{c} \\
\mathbf{b}^{*} & =\frac{2 \pi}{v_{0}} \mathbf{c} \wedge \mathbf{a} \\
\mathbf{c}^{*} & =\frac{2 \pi}{v_{0}} \mathbf{a} \wedge \mathbf{b}
\end{aligned}
$$

Where $v_{0}$ is the volume of the unit cell, $v_{0}=\mathbf{a} \cdot(\mathbf{b} \times \mathbf{c})$. A reciprocal lattice vector $\boldsymbol{\tau}$ connects the origin to a given node in reciprocal space

$$
\tau=h \mathbf{a}^{*}+k \mathbf{b}^{*}+l \mathbf{c}^{*}
$$

when $h, k$, and $l$ are integer numbers.

\section{Propagation vector and its star}

\subsection{Description of moments}

Before we detail what a magnetic structure is, we must begin with a description of the magnetic moment itself. There are of course a variety of ways and coordinate systems that can be used to describe a magnetic moment, e.g. Cartesian, polar or crystallographic coordinates. While it is of course preferable to describe the particular properties of the final structure in the most useful system (e.g. a rotation of a moment away from an axis is best described in terms of an angle), in the general case it is easiest to describe a moment in terms of projections along the crystallographic axes. Rather than say that a moment is of unit length and makes an angle of $0^{\circ}$ with the $c$-axis, we will simply say that the projection of the moments along the crystallographic axes can be described by a 'basis vector' $\Psi$ which has components along these axes. In this case, the basis vector is $\Psi=(001)$. In fact, when the basis vector is real, it simply corresponds to the projection of the moment along the different crystallographic axes, and so:

$$
\mathrm{m}_{j}=\Psi_{j}
$$

Often, however, the projections of the moment are described not just by one basis vector, but by the summation of several (see Section 5):

$$
\Psi_{j}=\sum_{\nu} C_{\nu} \psi_{\nu}
$$

In this work we will use $\psi_{\nu}$ to represent the $\nu$ components of $\Psi_{j}$ for a given propagation vector $\mathbf{k}$. The values of $\Psi_{j}$ will be taken as being those of atom $j$ in the zeroth unit cell (i.e. the crystallographic cell). 


\subsection{Formalism of a propagation vector $\mathrm{k}$}

Magnetic structures can be described by the periodic repetition of a magnetic unit cell, just as crystal structures are described by translation of a nuclear unit cell. For convenience, rather than building a complete magnetic unit cell (which could contain thousands of magnetic atoms) we use a description based on the nuclear unit cell and a 'propagation vector', $\mathbf{k}$, that describes the relation between moment orientations of equivalent magnetic atoms in different nuclear unit cells. This provides a simple and a general formalism for the description of a magnetic structure.

We illustrate this for the moment distribution $m_{j}$ associated with the atom $j$ of a magnetic structure. This can be Fourier expanded, whatever the nature of the ordering, according to:

$$
\mathbf{m}_{j}=\sum_{k} \boldsymbol{\Psi}_{j}^{\mathbf{k}} e^{-2 \pi i \mathbf{k} \cdot \mathbf{t}}
$$

That the summation is made over several wave vectors that are confined to the first Brillouin zone of the Bravais lattice of the nuclear cell is explained in detail in Section 3.4. If only one wave vector is involved, this simplifies to:

$$
\mathbf{m}_{j}=\Psi_{j}^{\mathbf{k}} e^{-2 \pi i \mathbf{k} \cdot \mathbf{t}}
$$

This equation describes the translation properties in real space of the basis vector $\boldsymbol{\Psi}_{j}$, which at present we can think of as the projections of the magnetic moment along the $a, b, c$ crystallographic axes with relation to the atomic site in the zeroth (nuclear) cell. At another atomic site (of the same type) in the crystal that is related by a lattice translation vector $t$, the projections of the moment on the 3 crystallographic axes are related to those in the nuclear cell by Equation 11. An example of this is shown in Figure 2. Here the magnetic unit cell is 2 times larger along the $c$-axis than the nuclear unit cell and the propagation vector is $k=00 \frac{1}{2}$. The moment in the zeroth cell is described by the basis vector $\Psi_{j}=\left(\begin{array}{ll}010\end{array}\right)$, that is to say the moment is pointing along the $b$-axis. When we move to the cell above (i.e. to a site that is related by the translation vector ${ }^{1} \mathrm{t}=\left(\begin{array}{lll}0 & 0 & 1\end{array}\right)$ the moment is rotated by $180^{\circ}$ and now points along the $(0-10)$ direction. As we move up the structure we find that the moment turns by $180^{\circ}$ for each nuclear cell translation until at $\mathrm{t}=\left(\begin{array}{lll}0 & 0 & 2\end{array}\right)$ it is the same as in the zeroth cell. In this way, if we know the basis vector that describes the moment orientation in the zeroth cell and the propagation vector, we can use Equation 11 to calculate the basis vector and moment orientation, of any equivalent atom in the crystal structure.

\subsection{Stability of magnetic structures}

When the sample is cooled and condenses into a state with magnetic order, the magnetic structure that results must leave the Hamiltonian invariant to lattice translations, i.e. the magnetic Hamiltonian of different unit cells must be the same. The minimisation in the magnetic energy of the system results in three possible situations:

- one $\mathbf{k}$ vector is more favourable than the others and the system chooses a ground state configuration that is described by:

$$
\mathbf{m}_{j}=\mathbf{\Psi}_{j}^{\mathbf{k}} e^{-2 \pi i \mathbf{k} \cdot \mathbf{t}}
$$

\footnotetext{
${ }^{1}$ Remember that a lattice translation vector in real space is given the symbol $t$, while one in reciprocal space has the symbol $\tau$.
} 


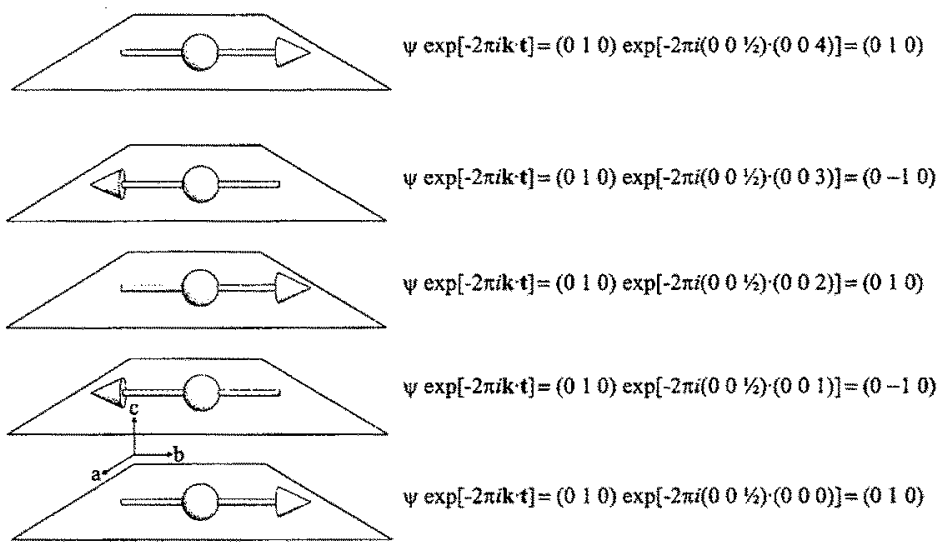

Figure 2: Description of translational properties with the propagation vector $\mathbf{k}$. In this example the basis vector for the moment in the zeroth cell is $\Psi=\left(\begin{array}{lll}0 & 1 & 0\end{array}\right), \mathrm{k}=00 \frac{1}{2}$ and each plane corresponds to a lattice translation of $t=001$

This is the most common situation and most of this work will be devoted to single $\mathbf{k}$ structures.

- several $\mathbf{k}$ vectors of the star are involved. The ground state is then described by:

$$
\mathbf{m}_{j}=\sum_{\mathbf{k}} \Psi_{j}^{k} e^{-2 \pi i \mathbf{k} \cdot \mathbf{t}}
$$

This is termed a 'multi-k' configuration.

- One $\mathbf{k}$ vector and its harmonics are involved, e.g. $\frac{k}{2}$. The ground state is then described by:

$$
\mathbf{m}_{j}=\sum_{\text {harmonics of } \mathbf{k}} \boldsymbol{\Psi}_{j}^{\mathbf{k}} e^{-2 \pi i \mathbf{k} \cdot \mathbf{t}}
$$

If the transition involves several arms of the star of propagation vector $\mathbf{k}$ and their harmonic terms, we have the possibility of crossed harmonics that are sometimes referred to as intermodulations.

\subsection{Star of the propagation vector $-\mathrm{k}$}

We will now consider the effects of the space group, $\mathrm{G}_{0}$, of our crystal structure on the propagation vector $\mathbf{k}$. For ease we will separate each symmetry element $g=\{h, \tau\}$ into rotation and translation parts, these are $h$ and $\tau$ respectively. The action of the rotation part $h$ on the reciprocal vector $\mathbf{k}$, results either in leaving $\mathbf{k}$ unchanged, or the generation of an unequivalent wave vector $\mathbf{k}^{\prime}$ :

$$
\mathbf{k}^{\prime}=\mathbf{k} h
$$



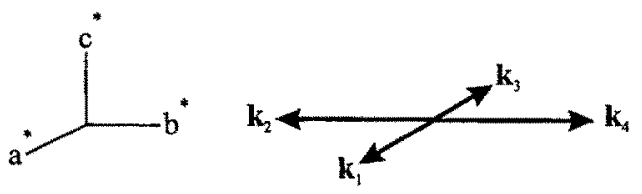

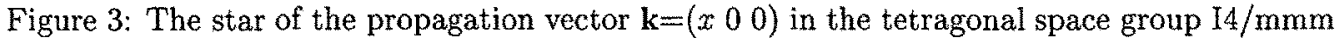
(point group $\left.D_{4 h}^{17}\right)$. The arms of the star are: $\mathbf{k}_{1}=\left(\begin{array}{lll}x & 0 & 0\end{array}\right), \mathbf{k}_{2}=\left(\begin{array}{lll}0 & -x & 0\end{array}\right), \mathbf{k}_{3}=\left(\begin{array}{lll}-x & 0 & 0\end{array}\right)$ and $\mathbf{k}_{4}=\left(\begin{array}{ll}0 \\ 0\end{array}\right.$ $x 0)$

where,

$$
\mathbf{k}^{\prime}=\mathbf{k} \quad \text { or } \quad \mathbf{k}^{\prime} \neq \mathbf{k}
$$

In the general case, a number of distinct propagation vectors will result from the operations of the rotational elements of the space group $G_{0}$ on the propagation vector $\mathbf{k}$. The symmetry elements of $G_{0}$ may then be classed into cosets, where the first coset $G_{k}$ is made up of elements that do not change the vector $k$, the second coset (given the symbol $g_{2}$ ) transform it into the unequivalent vector $\mathbf{k}_{2}$, and so on. If $g_{L}$ represents the elements of the coset $L$, we can write this relation as

$$
\mathbf{k}_{L}=\mathbf{k} g_{L}
$$

In this way, we find that the rotation elements of the space group $G_{0}$ gives rise to a set of unequivalent wave vectors. These we describe as being the 'star' of the propagation vector $\mathbf{k} ;$; 1$]$ each wave vector is an 'arm' of the star (and example of a star is given in Figure 3). The number of arms, $l_{\mathrm{k}}$, that make up a star is of course equal to the number of cosets and cannot exceed the number of elements in $\mathrm{G}_{0}$.

If Crystal Electric Field (CEF) or higher-order exchange interactions (e.g. quadrupolartype) are appropriate, several arms of a star can be involved in the structure, it is then said to be a 'multi-k' structure (this notion will be expanded upon in Section 4). More often, the magnetic structure is the result of only the first $\mathbf{k}$ vector. For this reason, we will now focus on the rotation elements that leave $\mathbf{k}$ invariant.

\subsection{The little group of the propagation vector $-\mathrm{k}$}

The symmetry elements of $\mathrm{G}_{0}$ that leave the $\mathbf{k}$ vector invariant are of particular importance in the determination of a magnetic structure. For this reason the elements of the first coset are given a special name- they make up the 'little group' $\mathrm{G}_{\mathbf{k}}$, and it is on these that all the Group Theory arguments that follow in Sections 7 and 8 are based. The little group will be discussed in greater detail in Section 8.

\section{Multi-domain and multi-k structures}

While the majority of magnetic structures that we come across involve only a single propagation vector $\mathbf{k}$, it is useful to see how the different types of propagation vectors can take part in a magnetic structure. Experimentally, these situations are revealed by the appearance of more than a single refection around a reciprocal lattice point. 

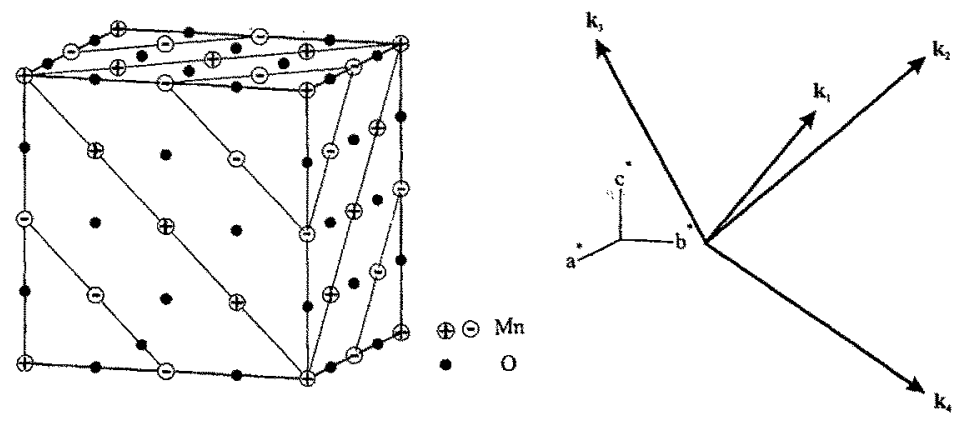

Figure 4: a) The magnetic motif of $\mathrm{MnO}$ made up of ferromagnetic planes of moments that are coupled antiferromagnetically. b) The star of $\mathbf{k}$ in reciprocal space is made up of the four propagation vectors related by the rotation elements of the space group $\mathrm{G}_{0}: \mathbf{k}_{1}=\left(\frac{1}{2}, \frac{1}{2}, \frac{1}{2}\right), \mathbf{k}_{2}=$ $\left(\frac{1}{2}, \frac{1}{2}, \frac{1}{2}\right), \mathbf{k}_{3}=\left(\frac{1}{2}, \frac{1}{2}, \frac{1}{2}\right)$ and $\mathbf{k}_{4}=\left(\frac{1}{2}, \frac{1}{2}, \frac{1}{2}\right)$. Domains are found that correspond to each of these k-vectors.

\subsection{Multi-domain structures}

The first magnetic neutron diffraction pattern collected was that of $\mathrm{MnO}$, published by Shull and Smart[2]. The period of the magnetic unit cell was found to be doubled along each of the cubic axes of the FCC structure, and so its volume is 8 times that of the crystallographic cell.

We now know that the structure in fact involves domains that order according to the 4 different arms of the propagation vector. The four $\mathrm{k}$ vectors involved are:

$$
\mathbf{k}_{1}=\left(\frac{1}{2}, \frac{1}{2}, \frac{1}{2}\right) \mathbf{k}_{2}=\left(\frac{1}{2}, \frac{1}{2}, \frac{1}{2}\right) \mathbf{k}_{3}=\left(\frac{1}{2}, \frac{1}{2}, \frac{1}{2}\right) \mathbf{k}_{4}=\left(\frac{1}{2}, \frac{1}{2}, \frac{1}{2}\right)
$$

As there are domains that order according to vector $\mathbf{k}_{1}$, others to $\mathbf{k}_{2}, \ldots, \mathbf{k}_{4}$, this is termed a 'multi-domain' structure.

Experimentally, different $\mathbf{k}$ domains will lead to different magnetic reflections, just as in multi- $k$ structures. In fact, the diffraction patterns of multiple domain and multi-k structures are identical and it is impossible to distinguish them without the application of an external constraint that breaks the symmetry on a macroscopic scale, and favours the population of one $\mathrm{k}$ domain over another.

\section{$4.2 \mathrm{k}$ and $-\mathrm{k}$ structures}

Structures that involve contributions from the two arms $\mathbf{k}$ and $\mathbf{- k}$ do not fall simply into the class of multi-k structures because, as we will show in Section 5, the requirement of a contribution from the $-\mathbf{k}$ arm can simply be the result of the form of the basis vectors, or the value of $\mathbf{k}$. Typically, the contribution of these two components gives rise to modulated magnetic structures, e.g. sine and ellipse structures.

\subsection{Multi -k structures}

As we have already seen, multi-k structures can involve different arms of the star of the propagation vector $\mathbf{k}$. This is a situation favoured by higher terms in the exchange Hamiltonian 


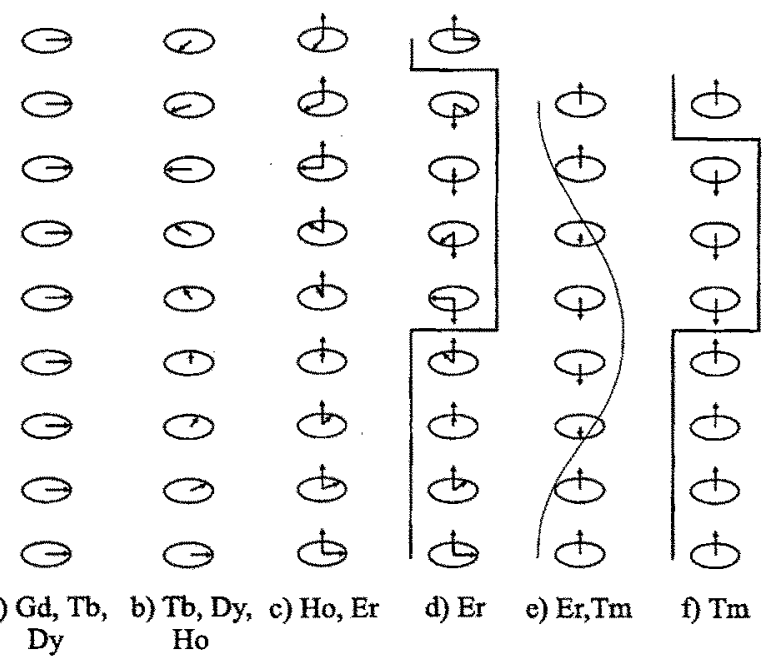

Figure 5: The magnetic structures of the heavy rare earth metals.

of the magnetic system. Also possible are structures that involve an 'accidental' degeneracy between the stars of unrelated propagation vectors. A magnetic transition that involves several stars does not necessarily follow the Landau theory for a second-order transition (Sections 7.3 and 7.2 ), but if suitable degeneracies occur the resulting structure may still order under a single Irreducible Representation.

\subsection{Structures that involve the harmonics of $\mathrm{k}$}

Addition of components of the harmonics of $\mathbf{k}$ to a structure will lead to a squaring up of the modulation, that is to say the magnitudes of the moments on the atoms becomes equal. This situation can be driven by CEF effects that disfavour any reduction in the amplitude of magnetic moment, or an instability of the modulated structure because of the large entropy associated with it. This is exemplified by a sine structure, where decreasing temperature leads to the structure becoming unstable and may lead to a squaring up of the modulation of the moments. Examples of this are the metals Er and Tm (see Figure 5) where third, fifth and higher order harmonics progressively appear with decreasing temperature. [3]

\section{Translation properties of magnetic structures}

Now that we will return to the situations that involve only a single propagation vector $\mathbf{k}$, and perhaps its inverse $-\mathbf{k}$. We have already shown how basis vectors and propagation vectors can be used in the description of magnetic structures. In this Section we will examine the different types of magnetic structures demonstrated in Figure 1. In the general case, the $\mathbf{k}$ vector may refer to any point within or on the surface of the first Brillouin zone. This gives rise to two general classes of magnetic structures: 
- Commensurate- the magnetic cell that is a simple multiple of the nuclear cell. It is in this group that are found the majority of known magnetic structures: simple ferromagnets, antiferromagnets and ferrimagnets.

- Incommensurate- there is no simple relation between the structural and magnetic cells.

It is important to note that these classifications describe only the propagation vector; the magnetic structure itself is the result of the propagation vector $\mathbf{k}$ and the basis vector $\Psi_{j}$. It is the combination of both of these that gives rise to the different possible structures $[1,4,5,6]$.

\subsection{Simple structures and Sine structures}

As we have seen, the translation properties of a magnetic structure may be described by:

$$
\mathrm{m}_{j}=\Psi_{j}^{\mathrm{k}} e^{-2 \pi i \mathbf{k} \cdot \mathbf{t}}
$$

Let us now expand the exponential:

$$
\mathbf{m}_{j}=\mathbf{\Psi}_{j}^{\mathrm{k}}[\cos (-2 \pi \mathbf{k} \cdot \mathbf{t})+i \sin (-2 \pi \mathbf{k} \cdot \mathbf{t})]
$$

and consider various possibilities for the basis vector $\Psi_{j}$ and the propagation vector $k$.

\subsection{1 is real and the sine component is null}

The simplest situation occurs when $\Psi_{j}^{\mathbf{k}}$ is a real basis vector. The condition that $\mathbf{m}_{j}$ is real requires that the sine component is zero- this occurs only for certain values of $\mathbf{k}$. Equation 20 then reduces to

$$
\mathbf{m}_{j}=\operatorname{Re}\left(\boldsymbol{\Psi}_{j}^{\mathbf{k}} \cos (-2 \pi \mathbf{k} \cdot \mathbf{t})\right)
$$

As the sine component is null, the cosine component is necessarily of maximal magnitude and so translation to another unit cell results only in some rotation of the moment, and does not change its magnitude. This is the situation in many simple ferromagnetic, ferrimagnetic, and antiferromagnetic structures (examples are given in Figure 1a-f).)

\subsection{2 $\Psi$ is real and the sine component is non-zero}

If the basis vector is real and the sine component is non-zero, Equation 20 leads to a magnetic moment that is complex- an impossible situation as the magnetic moment is a real entity. We are therefore left with the problem of how to relate our complex basis vector to the projections of a real moment. This in fact turns out to be very simple: the moment here cannot be described by a single propagation vector, but rather is described by contributions from 2 propagation vectors. The second propagation vector that is required in order to describe the magnetic moment distribution is the propagation vector $-\mathbf{k}$.

The atomic vector for an atom in the $n$th cell related to that in the zeroth cell by translation $\mathbf{t}$ is then given by: $[4]$

Where [4],

$$
\mathbf{m}_{j}=\Psi_{j}^{\mathrm{k}} e^{-2 \pi i \mathbf{k} \cdot \mathbf{t}}+\boldsymbol{\Psi}_{j}^{-\mathbf{k}} e^{2 \pi i \mathbf{k} \cdot \mathbf{t}}
$$




$$
\Psi_{j}^{-k}=\Psi_{j}^{\mathbf{k}^{*}}
$$

Insertion of this relation into Equation 22 and expansion of the exponential leads to

$$
\mathbf{m}_{j}=2 \operatorname{Re}\left(\Psi_{j}^{\mathbf{k}}\right) \cos (-2 \pi \mathbf{k} \cdot \mathbf{t})+2 \operatorname{Im}\left(\Psi_{j}^{\mathbf{k}}\right) \sin (-2 \pi \mathbf{k} \cdot \mathbf{t})
$$

As we are considering real basis vectors, the imaginary component in Equation 24 is zero and this reduces to

$$
\mathbf{m}_{j}=2 \operatorname{Re}\left(\boldsymbol{\Psi}_{j}^{\mathrm{k}}\right) \cos (-2 \pi \mathbf{k} \cdot \mathbf{t})
$$

We therefore see that if the propagation vector $\mathbf{k}$ leads a non-zero sine component in Equation 20 , the magnetic structure involves both the wave vectors $\mathbf{k}$ and $\mathbf{- k}$. A non-zero sine component requires also that the magnitude of the moment changes with translation through the crystal. The resulting structure has a sine modulation and an example is shown in Figure $1 \mathrm{~h}$.

\subsection{Helical structures}

\subsection{1 $\Psi$ is complex and $\operatorname{Re}(\Psi)=\operatorname{Im}(\Psi)$}

A complex basis vector associated with the vector $\mathbf{k}$ requires also a contribution from the $-\mathbf{k}$. Therefore, we begin again from Equation 24:

$$
\mathbf{m}_{j}=2 \operatorname{Re}\left(\Psi_{j}^{\mathbf{k}}\right) \cos (-2 \pi \mathbf{k} \cdot \mathbf{t})+2 \operatorname{Im}\left(\Psi_{j}^{\mathbf{k}}\right) \sin (-2 \pi \mathbf{k} \cdot \mathbf{t})
$$

If the real and imaginary components of $\Psi$ are equal we find that this simplifies to:

$$
\mathbf{m}_{j}=2 \operatorname{Re}\left(\boldsymbol{\Psi}_{j}^{\mathrm{k}}\right)[\cos (-2 \pi \mathbf{k} \cdot \mathbf{t})+\sin (-2 \pi \mathbf{k} \cdot \mathbf{t})]
$$

As the sine and cosine components define the points on a circle, the resulting structure is said to be a 'circular helix', i.e. one in which the magnitude of the moment is constant, but its orientation changes (Figure 1i).

\subsection{2 $\Psi$ is complex and $\operatorname{Re}(\Psi) \neq \operatorname{Im}(\Psi)$}

As the real and imaginary components are of different size, the equation

$$
\mathbf{m}_{j}=2 \operatorname{Re}\left(\boldsymbol{\Psi}_{j}^{\mathbf{k}}\right) \cos (-2 \pi \mathbf{k} \cdot \mathbf{t})+2 \operatorname{Im}\left(\boldsymbol{\Psi}_{j}^{\mathbf{k}}\right) \sin (-2 \pi \mathbf{k} \cdot \mathbf{t})
$$

describes an ellipse rather than a circle. The resulting structure is referred to as an 'elliptical helix' (Figure 1j).

\subsection{Summary of structures and basis vectors}

In this Section we have shown that the class of a magnetic structure is the result of both the propagation vector and the form of the basis vectors involved. Sine structures and simple structures arise from real basis vectors, while helices involve complex basis vectors. The key equations are

$$
\mathbf{m}_{j}=\Psi_{j}^{\mathbf{k}} e^{-2 \pi i k \cdot t}
$$

and 


$$
\mathbf{m}_{j}=2 \operatorname{Re}\left(\Psi_{j}^{\mathbf{k}}\right) \cos (-2 \pi \mathbf{k} \cdot \mathbf{t})+2 \operatorname{Im}\left(\Psi_{j}^{\mathbf{k}}\right) \sin (-2 \pi \mathbf{k} \cdot \mathbf{t})
$$

The calculation of these basis vectors will be detailed later in the section on Group Theory calculations (Section 8).

\section{Location of magnetic reflections}

\section{1 $k=0$ ferromagnetic (ferri- antiferromagnetic)}

As the magnetic and crystallographic unit cells are of the same size, the magnetic reflections occur at the nodes of the muclear reciprocal lattice and their intensities therefore add to those of the nuclear reflections. Assuming an unpolarised incident beam, the magnetic cross section[7, 8] is then given in barns by:

$$
\left(\frac{d \sigma}{d \Omega}\right)_{m a g}=\sum_{\tau}\left|\mathbf{M}_{\perp}(\mathbf{Q})\right|^{2} \delta(\mathbf{Q}-\tau)
$$

Where $\tau$ is a reciprocal space vector as defined by Equation 7 and $M_{\perp}(Q)$ is the magnetic interaction vector (the component of the magnetic structure factor perpendicular to the scattering vector $\mathbf{Q}$, in units of $\left.10^{-12} \mathrm{~cm}\right)$ :

$$
\mathbf{M}_{\perp}(\mathbf{Q})=0.269510^{-12} \mathbf{F}_{M \perp}(\mathbf{Q})
$$

where $F_{M \perp}(\mathbf{Q})$ has units of Bohr magnetons.

\section{2 $k \neq 0$ antiferromagnetic- commensurate}

The example shown in Figure $6 \mathrm{~b}$ is of the propagation vector $\mathrm{k}=\left(\frac{1}{2} 00\right)$. As the magnetic unit cell is 2 times larger in the $a$ direction than the nuclear cell, the reflections will occur at half-integer positions $\left(\frac{h}{2} k l\right)$.

\section{3 $\mathrm{k} \neq 0$ antiferromagnetic- incommensurate}

We know that due to the form of Equation 11, contributions from the basis vectors of both $\mathbf{k}$ and $-\mathbf{k}$ are required. Reflections will therefore be at positions associated with both of these propagation vectors, and pairs of Bragg reflections will surround each reciprocal lattice point. As demonstrated in Figure 6c, magnetic reflections will be observed at:

1. For $\mathbf{k}$

$$
\mathrm{Q}=\boldsymbol{\tau}+\mathbf{k}: \text { reflection } \tau^{+} \text {or }(\mathrm{hkl})^{+}
$$

2. For $-\mathbf{k}$

$$
\mathrm{Q}=\tau-\mathrm{k}: \text { reflection } \tau^{-} \text {or }(\mathrm{hkl})^{-}
$$


a) $k=0$

ferromagnetism

(ferri- or antiferromagnetism

in non-primitive cell)

$\left(\frac{d \sigma}{d \Omega}\right)_{M}=\sum_{\tau}\left|F_{M \perp}(Q)\right|^{2} \delta(Q-\tau)$

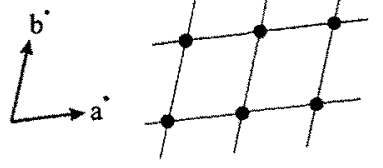

b) $k=\frac{1}{2} 00$

antiferromagnetism

(commensurate propagation vector)

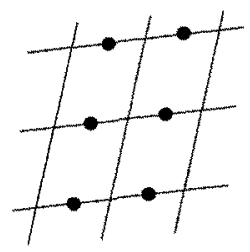

c) $\mathbf{k}=\mathrm{k}_{\mathrm{x}} \mathrm{k}_{\mathrm{y}} \mathrm{k}_{\mathrm{z}}$

antiferromagnetism

(incommensurate propagation vector.)

Contributions from both

$\mathrm{k}$ and $\mathbf{k}$ are required.

$\mathbf{k} \Rightarrow \mathbf{Q}=\tau+\mathbf{k}$

$-\mathbf{k} \Rightarrow \mathbf{Q}=\tau-\mathbf{k}$

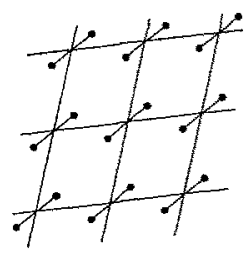

d) multi-k: $k_{x} k_{y} k_{z}+R_{1}\left(k_{x} k_{y} k_{z}\right)+\ldots$

antiferromagnetism

(incommensurate propagation vector.)

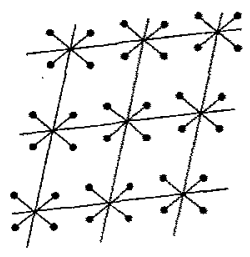

e) $\mathbf{k}$ with harmonics

antiferromagnetism

(incommensurate propagation vector.)

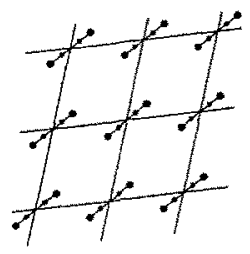

Figure 6: Cross-sections and graphs in reciprocal space for a variety of magnetic structure classes 


\subsection{Multi-k}

Figure $6 \mathrm{~d}$ demonstrates the diffraction pattern of a structure described by two incommensurate propagation vectors. We see that there is a pair of reflections for each propagation vector about the reciprocal lattice points. This magnetic pattern is exactly the same as that from a structure with two equally populated $\mathbf{k}$ domains and only the application of a suitable external constraint can allow the distinction of these situations.

\subsection{Harmonics of $\mathrm{k}$}

Contributions from the harmonics of $\mathbf{k}$ lead to the occurrence of reflections at positions that correspond to fractions of $\mathbf{k}$. The example given in Figure 6 is of an incommensurate propagation vector and its harmonic $\frac{1}{2} \mathbf{k}$.

\section{$7 \quad$ Symmetry in magnetic structures}

\subsection{The little group $\mathbf{G}_{\mathbf{k}}$ and its irreducible representations}

As we have already stated, the little group $G_{\mathbf{k}}$ that is made up of all the symmetry elements that leave $\mathbf{k}$ invariant, is a central concept in the symmetry analysis of magnetic structures. For a magnetic structure to be possible, it must be compatible with all of the symmetry operations of $\mathrm{G}_{\mathrm{k}}$ simultaneously. The set of matrices that describes how the moments transform under all of the operations of $G_{k}$ makes up a 'representation'. It is useful to separate these representations into orthogonal Irreducible Representations[9] (IRs), just as we separate the vibrations of a molecule into normal modes.

\subsection{Landau Theory and its application to magnetic phase transitions and structures}

The power and utility of Group Theory calculations with regards to the determination of magnetic structures comes from the Landau theory of a second-order phase transition. In the simplest of terms, this states that a second-order transition can involve the build up of magnetic fluctuations that have the symmetry of only one Irreducible Representation (in this case an Irreducible Representation describes the symmetry properties of a magnetic moment under all the symmetry operations of the little group $\left.\mathrm{G}_{\mathbf{k}}\right)[4,6,9]$. Because of this, the resulting magnetic structure can be described by the basis vectors associated with only that Irreducible Representation and the basis vectors associated with the Irreducible Representations not involved in the transition are necessarily zero. This greatly limits the number of possible magnetic models and the number of parameters that are involved in their refinement

Even in the cases where the transition is not second-order, nature is often kind to us and the structures that result are often the same as would be predicted for a second-order transition. The calculations detailed in Section 8 therefore continue to constitute a useful step in the determination, and description, of a magnetic structure.

\subsection{Application to structures with several magnetic sites}

If the unit cell of interest has several magnetic sites we have to consider how they will behave. If there are two types of site, $\mathrm{A}$ and $\mathrm{B}$, there are 3 limiting cases and we will consider each 
separately[4]:

- The two intra-site interactions are dominant: $I_{A}>I_{B}>I_{A B}$.

Here the coupling between the sites is small and so the sites behave independently. Each will therefore have its own ordering transition and no relation between the different Irreducible Representations involved is necessary.

- The inter-site interactions are dominant: $I_{A B}>I_{A}>I_{B}$.

The strong coupling between the sites leads to a single critical temperature. The basis vectors that are associated with both sites must belong to the same Irreducible Representation. This places a great restriction on the number of possible structures.

- One intra-site interaction is dominant: $I_{A}>I_{A B}>I_{B}$.

Upon cooling 2 distinct phase transitions will occur. The first involves the moments on the A sites. The inter-site coupling will lead to this structure polarising the $B$ moments. These will then display the same magnetic structure as the $A$ atoms. At a lower temperature, the $\mathrm{B}$ moments will undergo a symmetry-breaking transition and order cooperatively. The strong coupling between the $\mathrm{A}$ and $\mathrm{B}$ sites requires that these two orderings involve the same Irreducible Representation.

As an example, let us consider a system where there are 4 possible Irreducible Representations:

- Site A: $1 \Gamma_{1}+0 \Gamma_{2}+1 \Gamma_{3}+1 \Gamma_{4}$

- Site $\mathrm{B}: 1 \Gamma_{1}+1 \Gamma_{2}+0 \Gamma_{3}+0 \Gamma_{4}$

We know that only non-zero Irreducible Representations (labelled $\Gamma$ ) can be responsible for a magnetic structure. We see immediately that not all the Irreducible Representations occur on the two sites, i.e. on site $\mathrm{A}, \Gamma_{2}$ is not involved. If site A orders separately, the resulting structure will correspond to either that of $\Gamma_{1}, \Gamma_{3}$ or $\Gamma_{4}$, that is to say there are 3 possible magnetic 'models'. Similarly site $B$ could order according to $\Gamma_{1}$ or $\Gamma_{2}$. If there is no coupling between the sites and each orders separately, there are no symmetry restrictions on the possible Irreducible Representations involved. The sites can therefore order according to any of their allowed Irreducible Representations. However, if the situation is such that both order together, the two sites must order under the same Irreducible Representation, and only the Irreducible Representation $\Gamma_{1}$ can lead to a magnetic structure. The determination of the magnetic structure is therefore greatly simplified, as it can only involve the basis vectors associated with $\Gamma_{1}$.

\section{Representational Analysis}

\subsection{Group Theory and magnetic structures}

In non-primitive cells we must also determine the relation between the different magnetic moments in the cell. This relation can be very difficult to derive and is often found by comparison with known magnetic structures, or by trial and error. Group Theory arguments allow us to calculate symmetry-allowed relations between the moments and to greatly simplify this process. The results of these calculations are precisely the basis vectors, that we have been using to describe the magnetic structures. 
The technique that will be presented in this work involving the application of Group Theory to magnetic structures is termed Representational Analysis $[10,1,11]$. The only pieces of information that are required for these calculations are the propagation vector $\mathbf{k}$, the crystallographic space group and the atomic coordinates of the magnetic atoms before the magnetic phase transition. Rather than simply detailing the calculations involved, their application to an example problem will be used.

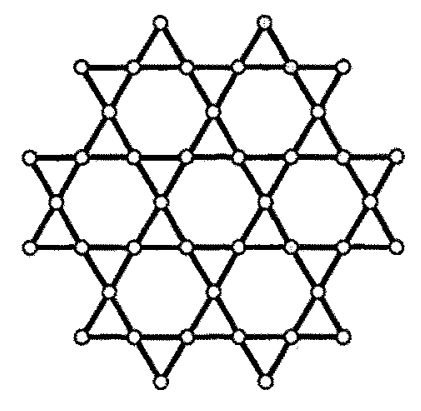

Figure 7: The kagomé lattice.

\subsection{Computer programs}

A number of computer programs exist that perform the calculations that make up magnetic symmetry analysis. Irreducible Representations can be calculated using KAREP[12], MODY[13], BASIREPS[14], and SARA $h[15]$. Basis vectors for the symmetry-allowed magnetic structures can be calculated using MODY, BASIREPS, or SARAh. All the Group Theory calculations and refinements presented here have been made using the program SARA $h$.

\subsection{Example: of $\mathrm{AgFe}_{3}\left(\mathrm{SO}_{4}\right)_{2}(\mathrm{OH})_{6}$ with $\mathrm{k}=00 \frac{3}{2}$}

The jarosites are described in the space group $R \overline{3} \mathrm{~m}$ (point group $D_{3 d}^{5}$ ) and their crystal structure is displayed in Figure 8. All the calculations that follow will refer to the hexagonal nonprimative setting of this space group, the symmetry elements of which are given in Table 1 . As the cell is hexagonal there are three kagome layers in the crystal structure and these have the stacking sequence ... $\mathrm{ABC}$.. The magnetic $\mathrm{Fe}^{3+}$ ions make up a 2-dimensional geometry called a kagomé lattice (Figure 7). In the mineral argento-jarosite, $\mathrm{AgFe}_{3}\left(\mathrm{SO}_{4}\right)_{2}(\mathrm{OH})_{6}$, the exchange is antiferromagnetic and magnetic ordering with a propagation vector $\mathrm{k}=00 \frac{3}{2}$ (with respect to the hexagonal axes) has been found at low temperature.[16, 17, 18] In this section we will calculate the symmetry-allowed magnetic structures using Representational Analysis. These calculations are also detailed in Ref. [17].

\subsection{The group $\mathrm{G}_{\mathrm{k}}$ and its Irreducible Representations}

As we have already explained in Section 3.4, for a given propagation vector $\mathbf{k}$, some of the operators of the space group $\mathrm{G}_{0}, g=\{h \mid \tau\}$, leave it invariant while others transform it into an equivalent vector that differs by some arbitrary translation of the reciprocal lattice, $\tau$, according to: 
Table 1: Symmetry elements of the space group $R \overline{3} \mathrm{~m}$. The notations used are of the International Tables, where the elements are separated into rotation and translation components, and the Jones faithful representations of the rotation parts. The latter corresponds to the vector formed from the operation of the rotation part of the element on $(x, y, z)$.

\begin{tabular}{|c|c|c|c|}
\hline Element number & IT notation & Jones symbol & Rotation matrix \\
\hline \multirow{4}{*}{ g1 } & & & 100 \\
\hline & $\left\{1 \mid \begin{array}{llll}1 & 0 & 0 & 0\end{array}\right\}$ & $x, y, z$ & $\begin{array}{lll}0 & 1 & 0\end{array}$ \\
\hline & & & $\begin{array}{lll}0 & 0 & 1\end{array}$ \\
\hline & & & $\begin{array}{lll}0 & 1 & 0\end{array}$ \\
\hline \multirow[t]{3}{*}{$\mathrm{g} 2$} & $\left\{3^{+} \mid 0000\right\}$ & $\bar{y}_{2} x-y, z$ & $1 \overline{1} 0$ \\
\hline & & & $\begin{array}{lll}0 & 0 & 1\end{array}$ \\
\hline & $\{3-1000\}$ & & $\begin{array}{lll}1 & 1 & 0 \\
1 & 0 & 0\end{array}$ \\
\hline \multirow{2}{*}{ g3 } & $\begin{array}{lll}10 & 0 & 0\end{array}$ & $y-x, x, 2$ & $\begin{array}{lll}1 & 0 & 0 \\
0 & 0 & 1\end{array}$ \\
\hline & & & $\begin{array}{lll}0 & 1 & 0\end{array}$ \\
\hline \multirow[t]{2}{*}{$\mathrm{g} 4$} & $\{2 \mid 0000\}$ & $y, x, \bar{z}$ & $\begin{array}{lll}1 & 0 & 0\end{array}$ \\
\hline & & & $\begin{array}{lll}0 & 0 & \overline{1} \\
1 & \overline{1} & 0\end{array}$ \\
\hline \multirow[t]{3}{*}{ g5 } & $\{2 \mid 000\}$ & $x-y, \bar{y}, \bar{z}$ & 0 I 0 \\
\hline & & & $\begin{array}{lll}0 & 0 & \overline{1}\end{array}$ \\
\hline & & & $\begin{array}{lll}\overline{1} & 0 & 0\end{array}$ \\
\hline \multirow[t]{2}{*}{ g6 } & $\{2 \mid 0000\}$ & $-x, y-x, \bar{z}$ & 110 \\
\hline & & & $\left(\begin{array}{lll}0 & 0 & 1 \\
1 & 0 & 0\end{array}\right.$ \\
\hline \multirow[t]{3}{*}{ g7 } & 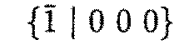 & $\bar{x}, \bar{y}, \bar{z}$ & $0 \quad \overline{1} 0$ \\
\hline & & & $\begin{array}{lll}0 & 0 & 1\end{array}$ \\
\hline & & & $\begin{array}{lll}0 & 1 & 0\end{array}$ \\
\hline \multirow[t]{2}{*}{$g 8$} & $\left\{\overline{3}^{+} \mid \begin{array}{lll}0 & 0 & 0\end{array}\right\}$ & $y, y-x, \bar{z}$ & $\begin{array}{lll}\overline{1} & 1 & 0\end{array}$ \\
\hline & & & $\begin{array}{lll}0 & 0 & 1 \\
1 & 1 & 0\end{array}$ \\
\hline \multirow[t]{2}{*}{ g9 } & 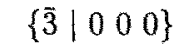 & $x-y, x, \bar{z}$ & $\begin{array}{lll}1 & 0 & 0\end{array}$ \\
\hline & & & $\begin{array}{lll}0 & 0 & \overline{1}\end{array}$ \\
\hline \multirow[t]{3}{*}{$\mathrm{g} 10$} & $\{\mathrm{~m} \mid 000\}$ & $\bar{y}, \bar{x}, z$ & $\begin{array}{lll}0 & 1 & 0 \\
1 & 0 & 0\end{array}$ \\
\hline & & & $\begin{array}{lll}0 & 0 & 1\end{array}$ \\
\hline & & & $\begin{array}{lll}\overline{1} & 1 & 0\end{array}$ \\
\hline \multirow[t]{2}{*}{$\mathrm{g} 11$} & $\{\mathrm{~m} \mid 0000\}$ & $y-x, y, z$ & $\begin{array}{lll}0 & 1 & 0\end{array}$ \\
\hline & & & $\begin{array}{lll}0 & 0 & 1 \\
1 & 0 & 0\end{array}$ \\
\hline \multirow[t]{2}{*}{ g12 } & $\left\{\mathrm{m} \mid \begin{array}{lll}0 & 0 & 0\end{array}\right\}$ & $x, x-y, z$ & $1 \overline{1} 0$ \\
\hline & & & $\left(\begin{array}{lll}0 & 0 & 1\end{array}\right.$ \\
\hline
\end{tabular}




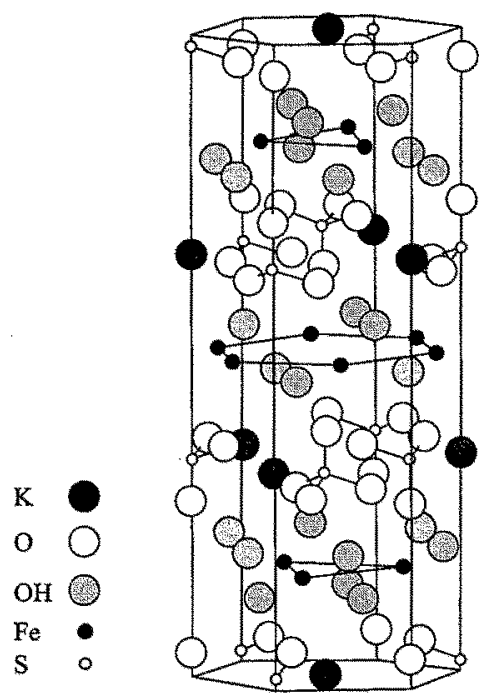

Figure 8: The jarosite crystal structure in the space group $R \overline{3} \mathrm{~m}$.

$$
\mathbf{k} h=\mathbf{k}+\boldsymbol{\tau}
$$

This set of elements makes up the so-called little group, $G_{k}$, which is a subgroup of $G_{0}$. The Irreducible Representations of this little group are given by the symbol $\Gamma_{\nu}$, where $\nu$ is the label of the irreducible representation, and the matrix that corresponds to the symmetry element $g$ is labelled by $d_{\nu}^{\mathbf{k}}(g)$

Table 2: First-order Irreducible Representations for the group $D_{3 d}^{5}$ for the vector $\mathrm{k}=00 \frac{3}{2}$.

\begin{tabular}{l|r|r|r|r|r|r|r|r|r|r|r|r} 
& $\mathrm{g} 1$ & $\mathrm{~g} 2$ & $\mathrm{~g} 3$ & $\mathrm{~g} 4$ & $\mathrm{~g} 5$ & $\mathrm{~g} 6$ & $\mathrm{~g} 7$ & $\mathrm{~g} 8$ & $\mathrm{~g} 9$ & $\mathrm{~g} 10$ & $\mathrm{~g} 11$ & $\mathrm{~g} 12$ \\
\hline$\Gamma_{1}$ & 1 & 1 & 1 & 1 & 1 & 1 & 1 & 1 & 1 & 1 & 1 & 1 \\
$\Gamma_{2}$ & 1 & 1 & 1 & 1 & 1 & 1 & -1 & -1 & -1 & -1 & -1 & -1 \\
$\Gamma_{3}$ & 1 & 1 & 1 & -1 & -1 & -1 & 1 & 1 & 1 & -1 & -1 & -1 \\
$\Gamma_{4}$ & 1 & 1 & 1 & -1 & -1 & -1 & -1 & -1 & -1 & 1 & 1 & 1
\end{tabular}

Looking at the example of $\mathrm{AgFe}_{3}\left(\mathrm{SO}_{4}\right)_{2}(\mathrm{OH})_{6}$ with $\mathrm{k}=00 \frac{3}{2}$, we find that the little group contains all of the 12 symmetry operators of the space group $R 3 \bar{m}$. The Irreducible Representations of these are given in Tables 2 and 3 . One sees immediately that the second-order representations $\Gamma_{5}$ and $\Gamma_{6}$ have the same elements for symmetry operations 1-6 and are related by a factor of $(-1)$ for the operations $7-12$.

These Irreducible Representations may be verified against tabulated values of the projective (or 'loaded') representations, $d_{\nu}^{p r}$, given in works such as Bradley and Cracknell[9] and Kovalev[19]. The tabulated representations are given for the various point group symmetries and can be converted into the Irreducible Representations of the little group $G_{k}$ of the propagation vector $\mathbf{k}$ by multiplicating them with a phase factor:

$$
d_{\nu}=d_{\nu}^{p r} \cdot \mathrm{e}^{-2 \pi \mathbf{k} \cdot \tau}
$$


Table 3: Second-order Irreducible Representations for the group $D_{3 d}^{5}$ for the vector $\mathbf{k}=00 \frac{3}{2}$. $\epsilon=\exp \left(-\frac{2 \pi}{3}\right)$.

\begin{tabular}{|c|c|c|c|c|c|c|}
\hline & g1 & $g 2$ & $\mathrm{~g} 3$ & $\mathrm{~g} 4$ & g5 & g6 \\
\hline \multirow{3}{*}{$\Gamma_{5}, \Gamma_{6}$} & 10 & $\epsilon \quad 0$ & $\epsilon^{2} \quad 0$ & 01 & $0 \epsilon^{2}$ & $0 \epsilon$ \\
\hline & $\begin{array}{ll}0 & 1\end{array}$ & $0 \quad \epsilon^{2}$ & $0 \epsilon$ & 10 & $\epsilon \quad 0$ & $\epsilon^{2} \quad 0$ \\
\hline & g7 & g8 & $\mathrm{g9}$ & 810 & g11 & g12 \\
\hline \multirow[b]{2}{*}{$\Gamma_{5}$} & $-1 \quad 0$ & $\begin{array}{ll}-\epsilon & 0\end{array}$ & $-\epsilon^{2}$ & $0-1$ & $0-\epsilon^{2}$ & $0-\epsilon$ \\
\hline & $\begin{array}{ll}0 & -1\end{array}$ & $0-\epsilon^{2}$ & $0-\epsilon$ & -1 & $-\epsilon$ & $-\epsilon^{2}$ \\
\hline \multirow{2}{*}{$\Gamma_{6}$} & 10 & $\epsilon \quad 0$ & $\begin{array}{ll}\epsilon^{2} & 0\end{array}$ & 01 & $0 \epsilon^{2}$ & \\
\hline & $\begin{array}{ll}0 & 1\end{array}$ & $0 \quad \epsilon^{2}$ & $0 \epsilon$ & 10 & $\begin{array}{ll}\epsilon & 0\end{array}$ & $\begin{array}{ll}\epsilon^{2} & 0\end{array}$ \\
\hline
\end{tabular}

Where $\tau$ represents the translation part of the symmetry operator to which $d_{\nu}$ is associated,

\subsection{Effect of symmetry element on a moment bearing atom}

The effect of a symmetry element is two-fold: it will act to change the position of an atom, and reorientate the magnetic moment, e.g. atom 1 moves to the position of atom 2, and its moment is reversed. The combination of these two results are described by the magnetic representation, $\Gamma$. We will examine these two effects separately:

\subsubsection{Effect of symmetry element on atom positions: the permutation represen- tation}

A symmetry operator $g=\{h \mid \tau\}$ acts on both the position $r_{j}$ of the atom and on the components $\alpha$ of the axial vector that describes the moment. The operation that sends $r_{j}$ in the zeroth cell to $r_{i}$ in the $p$ th cell can be symbolically stated as

$$
g(j 0) \rightarrow\left(i a_{p}\right)
$$

In other terms, the effect of a symmetry operation $g$ is to permute the column matrix of atom labels, $\mathbf{P}$ :

$$
g(\mathbf{P}) \rightarrow \mathbf{P}^{\prime}
$$

This operation is governed by a permutation representation, $\Gamma_{\text {perm }}$, which has matrices of order $\mathrm{N}_{A}$, where $\mathrm{N}_{A}$ is the number of equivalent positions of the crystallographic site. It is important to note that when a symmetry operation results in an atomic position that is outside the zeroth cell, a phase factor must be included that relates the generated position to that in the zeroth cell. This phase is simply given by:

$$
\theta=-2 \pi \mathbf{k} \cdot \mathbf{T}
$$

Where $T$ is the translation vector, that relates the original and generated atoms.

As an example, from Table 4 we see that the permutation equation for the atoms of the three Bravais sublattices under the $g=\left\{3^{+} \mid \begin{array}{lll}0 & 0 & 0\end{array}\right.$ operation is:

$$
\left(\begin{array}{c}
2 \cdot \exp \left(\theta_{a}\right) \\
3 \cdot \exp \left(\theta_{b}\right) \\
1 \cdot \exp \left(\theta_{c}\right)
\end{array}\right)=\Gamma_{p e r m}\left(\begin{array}{l}
1 \\
2 \\
3
\end{array}\right)
$$


Where the atomic positions follow the labelling: $1=\left(\begin{array}{lll}\frac{1}{2} & \frac{1}{2} & \frac{1}{2}\end{array}\right), 2=\left(\begin{array}{lll}\frac{1}{2} & 0 & \frac{1}{2}\end{array}\right), 3=\left(\begin{array}{lll}0 & \frac{1}{2} & \frac{1}{2}\end{array}\right)$. For the operation $g=\left\{3^{+} \mid 000\right\}, \theta_{a}=\theta_{b}=\theta_{c}=0$ for $\mathbf{k}=00 \frac{3}{2}$.

The permutation representation is therefore given by

$$
\Gamma_{\text {perm }}^{\{3+\mid 000\}}=\left(\begin{array}{lll}
0 & 1 & 0 \\
0 & 0 & 1 \\
1 & 0 & 0
\end{array}\right)
$$

The character of this representation, $\chi_{\text {perm }}$, for each symmetry operator is then simply the sum of the phases $\theta(g)$ for the atoms that are transformed into an equivalent atom under a symmetry operation, and so for both the propagation vectors, $\chi_{p e r m}^{\left\{3^{+} \mid 000\right\}}=0$.

\subsubsection{Effect of symmetry element on moment vectors: the axial vector represen- tation}

The second effect of this symmetry operation is to transform the spin components with index $\alpha,(\alpha=x, y, z)$ of the reference spin $j$ into the index $\alpha^{\prime}$ of the atom at $r_{i} \cdot[10,1,11]$ These transformations are described by the axial vector representation, $\tilde{V}$, the character of which is given by

$$
\chi_{\tilde{V}}^{h}=\sum_{a=b} R_{a b}^{h} \operatorname{det}(h),
$$

Where $R_{a b}^{h}$ refers to a specific element $a, b$ of the rotation matrix $h$, and $\operatorname{det}(h)$ represents the determinant of the rotation matrix $R^{h}$, and has the value of +1 for a proper and -1 for an improper rotation. This is exemplified for the $3^{+}$rotation, where the operation of $h\left(3^{+}\right)$on the moment vector $\vec{M}=\left(\mathrm{m}_{x}, \mathrm{~m}_{y} \mathrm{~m}_{z}\right)$ gives:

$$
\begin{aligned}
R\left(3^{+}\right) \vec{M} & =\operatorname{det}(h)\left(\begin{array}{ccc}
0 & \overline{1} & 0 \\
1 & \overrightarrow{1} & 0 \\
0 & 0 & 1
\end{array}\right)\left(\begin{array}{l}
m_{x} \\
m_{y} \\
m_{z}
\end{array}\right) \\
& =1 \quad\left(\begin{array}{c}
-m_{y} \\
m_{x}-m_{y} \\
m_{z}
\end{array}\right)
\end{aligned}
$$

As $3^{+}$is a proper rotation, $\operatorname{det}\left(3^{+}\right)=1$ and the character of $\tilde{V}$ for $\mathrm{h}\left(3^{+}\right)$is therefore $\chi_{\vec{V}}^{3^{+}}=0$.

\subsection{Magnetic representation}

As we have already stated, the magnetic representation, $\Gamma$, describes both the result of the symmetry operation on the atomic positions, and on the axial vectors that describe the magnetic moments. As these effects are independent, the magnetic representation is given by their direct product $[10,11,1]$ :

$$
\Gamma=\tilde{V} \times \Gamma_{\text {Perm }}
$$

Or, in terms of the matrices for the representations themselves

$$
D_{\left(h, \boldsymbol{\tau}_{h}\right)}^{\Gamma}=D_{(h)}^{\tilde{V}} \times D_{\left(h, \boldsymbol{\tau}_{h}\right)}^{\Gamma_{P_{e r m}}}
$$


Table 4: The permutation of $\mathrm{B}^{3+}$ atoms (at position 9d) and the transformation of the axial components of the moment under the different symmetry operators of the $R \overline{3} \mathrm{~m}$ space group (point group $D_{3 d}^{5}$ ) for $\mathbf{k}=00 \frac{3}{2}$. The characters of the representations $\Gamma_{\text {perm }}$ and $\tilde{V}$ are given.

\begin{tabular}{|c|c|c|c|c|c|c|c|c|c|}
\hline \multirow{2}{*}{\multicolumn{2}{|c|}{$g=\{h \mid \tau\}$}} & \multicolumn{3}{|c|}{ Atoms } & \multirow[b]{2}{*}{$\chi_{\text {perm }}$} & \multicolumn{4}{|c|}{ Axial vector components } \\
\hline & & 1 & 2 & 3 & & $m_{x}$ & $\mathrm{~m}_{y}$ & $\mathrm{~m}_{z}$ & $\chi_{\hat{V}}$ \\
\hline$\{1$ & $\begin{array}{lll}0 & 0 & 0\end{array}$ & 1 & 2 & 3 & 3 & $\mathrm{~m}_{x}$ & $\mathrm{~m}_{y}$ & $\mathrm{~m}_{z}$ & 8 \\
\hline$\left\{3^{+}\right.$ & $\left.\begin{array}{lll}0 & 0 & 0\end{array}\right\}$ & 2 & 3 & 1 & 0 & $-\mathrm{m}_{y}$ & $\mathrm{~m}_{x}-\mathrm{m}_{y}$ & $\mathrm{~m}_{z}$ & 0 \\
\hline$\left\{3^{-}\right.$ & $\begin{array}{lll}0 & 0 & 0\end{array}$ & 3 & 1 & 2 & 0 & $-\mathrm{m}_{x}+\mathrm{m}_{y}$ & $-m_{x}$ & $\mathrm{~m}_{z}$ & 0 \\
\hline$\{2$ & 0 & -1 & -3 & -2 & -1 & $\mathrm{~m}_{y}$ & $\mathrm{~m}_{x}$ & $-\mathrm{m}_{z}$ & -1 \\
\hline$\{2$ & $\left.\begin{array}{lll}0 & 0 & 0\end{array}\right\}$ & -3 & -2 & -1 & -1 & $\mathrm{~m}_{x}-\mathrm{m}_{y}$ & $-\mathrm{m}_{y}$ & $-m_{z}$ & -1 \\
\hline$\{2$ & $\left.\begin{array}{lll}0 & 0 & 0\end{array}\right\}$ & -2 & -1 & -3 & -1 & $-\mathrm{m}_{x}$ & $-m_{x}+m_{y}$ & $-\mathrm{m}_{z}$ & -1 \\
\hline$\{\overline{1}$ & $\begin{array}{lll}0 & 0 & 0\end{array}$ & -1 & -2 & -3 & -3 & $\mathrm{~m}_{x}$ & $\mathrm{~m}_{y}$ & $\mathrm{~m}_{z}$ & -3 \\
\hline$\left\{\overline{3}^{+}\right.$ & $\left.\begin{array}{llll}0 & 0 & 0\end{array}\right\}$ & -2 & -3 & -1 & 0 & $-m_{y}$ & $m_{x}-m_{y}$ & $m_{z}$ & 0 \\
\hline$\{\overline{3}$ & $\left.\begin{array}{lll}0 & 0 & 0\end{array}\right\}$ & -3 & -1 & -2 & 0 & $-m_{x}+m_{y}$ & $-\mathrm{m}_{x}$ & $\mathrm{~m}_{z}$ & 0 \\
\hline$\{\mathrm{m}$ & $\left.\begin{array}{lll}0 & 0 & 0\end{array}\right\}$ & 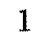 & 3 & 2 & 1 & $\mathrm{~m}_{y}$ & $m_{x}$ & $-m_{z}$ & -1 \\
\hline$\{\mathrm{m}$ & $\left.\begin{array}{lll}0 & 0 & 0\end{array}\right\}$ & 3 & 2 & 1 & 1 & $\mathrm{~m}_{x}-\mathrm{m}_{y}$ & $-m_{y}$ & $-m_{z}$ & -1 \\
\hline$\{\mathrm{m}$ & $\begin{array}{lll}0 & 0 & 0\end{array}$ & 2 & 1 & 3 & 1 & $-m_{x}$ & $-m_{x}+m_{y}$ & $-\mathrm{m}_{z}$ & -1 \\
\hline
\end{tabular}

The characters of these representations are related according to:

$$
\chi_{\Gamma}=\chi_{\tilde{V}} \times \chi_{\text {perm }}
$$

\subsection{Reduction of the Representation $\Gamma$}

The magnetic representation for a particular site can be decomposed into contributions from the Irreducible Representations of the little group:

$$
\Gamma=\sum_{\nu} n_{\nu} \Gamma_{\nu}
$$

where $\mathrm{n}_{\nu}$ is the number of times the irreducible representation $\Gamma_{\nu}$ appears in the magnetic representation $\Gamma . n_{\nu}$ is given by:

$$
n_{\nu}=\frac{1}{n\left(G_{k}\right)} \sum_{h \in G_{k}} \chi_{\Gamma}(h) \chi_{\Gamma_{\nu}^{*}}(h)
$$

Here, $\chi_{\Gamma}$ is the character of the magnetic space group and $\chi_{\Gamma_{\nu}^{*}}$ is the complex conjugate of the character of the irreducible representation with index $\nu$.

\subsection{Calculation of the basis vectors $\Psi$}

The basis vectors, $\psi_{n}$, that transform according to the $\mu$ dimensional irreducible representation $\Gamma_{\nu}^{(\mu)}$ are projected out of the representation matrix $D_{\nu}$ using a series of test functions $\phi^{\beta}$, where $\phi^{1}=(100), \phi^{2}=(010)$, and $\phi^{3}=(001)$. This is carried out by the projection operator formula:

$$
\psi_{n}^{i \lambda}=\sum_{g \in G_{\mathbf{k}}} D_{\nu}^{* \lambda}(g) \delta_{i, g i} \mathrm{e}^{-2 \pi i \mathbf{k} \cdot\left(\mathbf{r}_{g i}-\mathbf{r}_{i}\right)} \operatorname{det}(h) R^{h} \phi^{\beta},
$$

The summation is over the symmetry elements of the little group $\mathrm{G}_{\mathbf{k}} . \psi$ is a spin component that we represent by a column matrix $\psi(\mathbf{r}) . \delta_{i, g_{i}}$ is unity if the atoms $i$ and $g i$ are equivalent 
positions of the crystallographic site that are related by a primitive lattice translation, i.e. they are of the same sublattice of the Wyckoff site. Equation 48 is applied sequentially to each element $\lambda$ of the matrix $D_{v}$, for each equivalent position $i$ of the crystallographic site. The row of the matrices $D_{\nu}$ is fixed during the examination of a given IR.

In our calculations the $\mu$ elements are those that correspond to the first row of the matrix of $D_{\nu}$. As for each element, labelled $\lambda=1 \ldots \mu$, three components $\beta$ are projected out, there are in total $3 \mu$ projected components. Of these, the number of non-zero unique projected components for a representation is of course the same as calculated using Equation (47).

\subsection{Refinement of basis vectors mixing coefficients}

Any linear combination of basis vectors within one representation is necessarily a symmetryallowed basis vector. The atomic moment on a particular atom, $\mathbf{m}_{j}$, is therefore most generally given by the sum of the basis vectors for a particular irreducible representation:

$$
\mathbf{m}_{j}=\sum_{\nu} C_{\nu} \psi_{\nu}
$$

where $C_{\nu}$ is the mixing coefficient of the basis vector $\nu$. In refining the orientation of an atomic moment, we are in effect refining the mixing coefficients $C_{\nu}$ of the basis vectors within the irreducible representation being examined. The number of variables in our refinement is simply the number of unique basis vectors that transform according to a given representation, i.e. $n_{\nu} \mu$.

\subsection{Refinement of complex basis vectors}

The refinement of the mixing coefficients that relate complex basis vectors will be dealt with in detail, to demonstrate how an ordered array of magnetic moments, which are necessarily real entities, can be described by complex basis vectors.

\subsection{Decomposition of the magnetic representation and the basis vectors of $\mathrm{AgFe}_{3}\left(\mathrm{SO}_{4}\right)_{2}(\mathrm{OH})_{6}$}

In the hexagonal setting the magnetic $\mathrm{Fe}^{3+}$ ions are found on the $9 \mathrm{~d}$ sites. For these sites the decomposition of the magnetic representation according to Equation 47 is:

$$
\Gamma=0 \Gamma_{1}^{(1)}+2 \Gamma_{2}^{(1)}+0 \Gamma_{3}^{(1)}+1 \Gamma_{4}^{(2)}+3 \Gamma_{5}^{(2)}+0 \Gamma_{6}^{(2)}
$$

The Landau theory of a second-order phase transition, requires that only one representation is involved, and so for this $\mathbf{k}$ there are only three possible magnetic structures. These correspond to representations $\Gamma_{2}, \Gamma_{4}$ and $\Gamma_{5}$.

The basis vectors for these representations calculated according to Equation 48 are given in Table 5. The atomic sites are labelled following the convention given in Section 8.5.1. The basis vectors have varied forms and we will now explain in detail the types of magnetic structures that they correspond to.

Representations $\Gamma_{2}$ and $\Gamma_{4}$ are one dimensional. They therefore correspond to simple magnetic structures in which the atomic moments are orientated along particular crystallographic axes. It is noteworthy that both $\psi_{1}$ and $\psi_{2}$ correspond to $120^{\circ}$ spin structures, with the total spin on any given triangle plaquette being $\sum_{i} S_{i}=0$. While, the two spin structures are in fact 
Table 5: The basis vectors of the Irreducible Group Representations of the space group $R \overline{3} \mathrm{~m}$ (point group $D_{3 d}^{5}$ ) appearing in the magnetic representation with $\mathbf{k}=00 \frac{3}{2}$

\begin{tabular}{c|c|ccc|ccc|ccc} 
IR & $\mathrm{b}-\mathrm{v}$ & \multicolumn{2}{|c|}{ Atom 1} & \multicolumn{4}{|c}{ Atom 2} & \multicolumn{3}{c}{ Atom 3} \\
& & $\mathrm{~m}_{x}$ & $\mathrm{~m}_{y}$ & $\mathrm{~m}_{z}$ & $\mathrm{~m}_{x}$ & $\mathrm{~m}_{y}$ & $\mathrm{~m}_{z}$ & $\mathrm{~m}_{x}$ & $\mathrm{~m}_{y}$ & $\mathrm{~m}_{z}$ \\
\hline$\Gamma_{2}$ & $\psi_{1}$ & 1 & -1 & 0 & 1 & 2 & 0 & -2 & -1 & 0 \\
& $\psi_{2}$ & 0 & 0 & 1 & 0 & 0 & 1 & 0 & 0 & 1 \\
\hline$\Gamma_{4}$ & $\psi_{3}$ & 1 & 1 & 0 & -1 & 0 & 0 & 0 & -1 & 0 \\
\hline$\Gamma_{5}$ & $\psi_{4}$ & 1 & 0 & 0 & 0 & $-\frac{1}{2}-\frac{\sqrt{3}}{2} \mathrm{i}$ & 0 & $\frac{1}{2}-\frac{\sqrt{3}}{2} \mathrm{i}$ & $\frac{1}{2}-\frac{\sqrt{3}}{2} \mathrm{i}$ & 0 \\
& $\psi_{5}$ & 0 & 1 & 0 & $\frac{1}{2}+\frac{\sqrt{3}}{2} \mathrm{i}$ & $\frac{1}{2}+\frac{\sqrt{3}}{2} \mathrm{i}$ & 0 & $-\frac{1}{2}+\frac{\sqrt{3}}{2} \mathrm{i}$ & 0 & 0 \\
& $\psi_{6}$ & 0 & 0 & 1 & 0 & 0 & $-\frac{1}{2}-\frac{\sqrt{3}}{2} \mathrm{i}$ & 0 & 0 & $-\frac{1}{2}+\frac{\sqrt{3}}{2} \mathrm{i}$ \\
& $\psi_{7}$ & 0 & 1 & 0 & $\frac{1}{2}-\frac{\sqrt{3}}{2} \mathrm{i}$ & $\frac{1}{2}-\frac{\sqrt{3}}{2} \mathrm{i}$ & 0 & $-\frac{1}{2}-\frac{\sqrt{3}}{2} \mathrm{i}$ & 0 & 0 \\
& $\psi_{8}$ & 1 & 0 & 0 & 0 & $-\frac{1}{2}+\frac{\sqrt{3}}{2} \mathrm{i}$ & 0 & $\frac{1}{2}+\frac{\sqrt{3}}{2} \mathrm{i}$ & $\frac{1}{2}+\frac{\sqrt{3}}{2} \mathrm{i}$ & 0 \\
& $\psi_{9}$ & 0 & 0 & -1 & 0 & 0 & $\frac{1}{2}-\frac{\sqrt{3}}{2} \mathrm{i}$ & 0 & 0 & $\frac{1}{2}+\frac{\sqrt{3}}{2} \mathrm{i}$
\end{tabular}

related by a global rotation of spins, the two representations differ in that $\Gamma_{2}$ allows the introduction of an out-of-plane component, which corresponds to $\psi_{2}$. The combination of the 2 basis functions $\psi_{1}$ and $\psi_{2}$ creates a so-called 'umbrella structure' (of the type shown in Figure 1f) in which the degree of out-of-plane canting is a refinement variable.

Representation $\Gamma_{5}$ is two dimensional and is repeated 3 times. It therefore corresponds to a 6 basis vector magnetic structure. As the general solution involves any linear combination of these 6 basis vectors we can not ascribe to this representation a simple structure. We do note however, that there are relations between the basis vectors and these will simplify the refinement of the mixing coefficients: $\psi_{4}^{*}=\psi_{8}, \psi_{5}^{*}=\psi_{7}$ and $\psi_{6}^{*}=-\psi_{9}$.

As the atomic spins are real entities, in the case of complex basis vectors it is necessary to introduce the corresponding basis vectors of the propagation vector $-\mathbf{k}$ in order to make the summation of the two components real (Section 5). A description of the translation properties of this structures begins as normal from:

$$
\mathbf{m}_{j}=\Psi_{j}^{\mathbf{k}} e^{-2 \pi i \mathbf{k} \cdot \mathbf{t}}+\Psi_{j}^{-\mathbf{k}} e^{2 \pi i \mathbf{k} \cdot \mathbf{t}}
$$

However, as for $\mathbf{k}=00 \frac{3}{2}$ the vectors $\mathbf{k}$ and $-\mathbf{k}$ are equivalent, we have $-\mathbf{k}=\mathbf{k}-3 \mathbf{c}^{*}$ and so

$$
e^{-2 \pi i k \cdot t}=e^{2 \pi i k \cdot t}
$$

and

$$
\mathbf{m}_{j}=\left(\boldsymbol{\Psi}_{j}^{\mathbf{k}}+\mathbf{\Psi}_{j}^{-\mathbf{k}}\right) e^{2 \pi i \mathbf{k} \cdot \mathbf{t}}
$$

A further simplification arises from the fact that the addition of the -k contribution corresponds to the addition of the conjugate of the basis vector of $\mathbf{k}$, i.e.

$$
\Psi_{j}^{-k}=\Psi_{j}^{k^{*}}
$$

We therefore obtain

$$
\mathbf{m}_{j}=2 \operatorname{Re}\left(\boldsymbol{\Psi}_{j}^{\mathbf{k}}\right)\left[\cos \left(2 \pi \mathbf{k} \cdot \mathbf{t}_{n}\right)+i \sin (2 \pi \mathbf{k} \cdot \mathbf{t})\right]
$$


For both $\mathbf{k}=00 \frac{3}{2}$ the sine component vanishes under the centring translations of the nonprimitive cell, or integer translations of the crystallographic cell, and so Equation 55 reduces to

$$
\mathbf{m}_{j}=2 \operatorname{Re}\left(\boldsymbol{\Psi}_{j}^{\mathrm{k}}\right) \cos (2 \pi \mathbf{k} \cdot \mathbf{t})
$$

As in general, the basis vectors associated with $\Gamma_{5}$ that are to be tested against the magnetic structure are complex, when considering the translational properties of the magnetic moments it is sufficient just to add their complex conjugate in order to arrive at real values for the atomic moments. In these two cases this leads to Equation 56.

An alternative way of dealing with complex basis vectors is to examine the properties of the basis vectors associated with an irreducible representation. In some cases ${ }^{2}$ by mixing the different basis vectors in particular ways, with perhaps the addition to all the basis vectors of a phase factor, real basis vectors can be defined. This technique corresponds to the transformation of the complex basis vector space into an equivalent real basis.
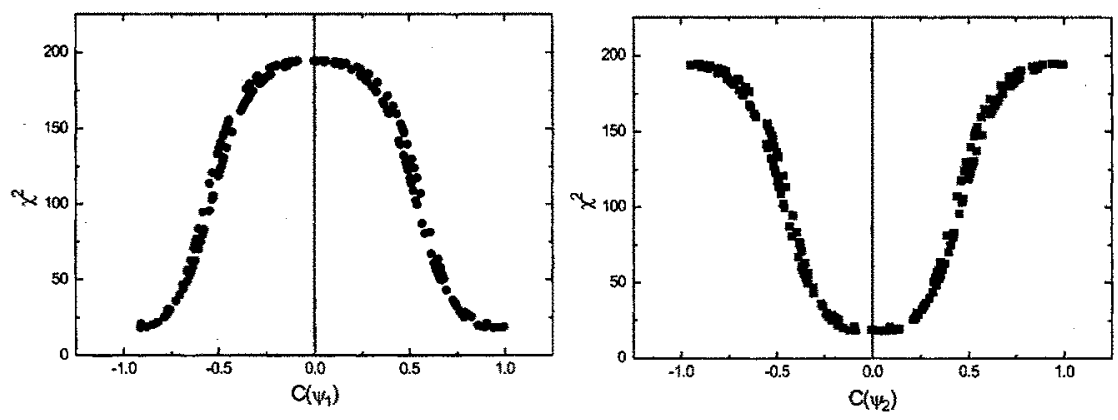

Figure 9: $\chi^{2}$ as a function of the basis vector coefficients $C\left(\boldsymbol{\psi}_{1}\right)$ and $C\left(\boldsymbol{\psi}_{2}\right)$ during the refinement of the magnetic structure of $\mathrm{AgFe}_{3}\left(\mathrm{SO}_{4}\right)_{2}(\mathrm{OD})_{6}$ at $1.5 \mathrm{~K}$.

\subsection{Refinement of the magnetic structure of $\mathrm{AgFe}_{3}\left(\mathrm{SO}_{4}\right)_{2}(\mathrm{OD})_{6}$}

The collected neutron diffraction data were found to be compatible only with a magnetic structure described by the representation $\Gamma_{4}$. Figure 9 displays the value of $\chi^{2}$ as a function of the mixing coefficients $C\left(\psi_{1}\right)$ and $C\left(\psi_{2}\right)$; the only refinement variable in the least-squares matrix was the magnitude of the magnetic moment. In all cases the sum of the mixing coefficients was adjusted to be unity, and a trivial factor was then used to separately scale the magnitudes of moments described. The best value of $\chi^{2}$ corresponds to the coefficients $C\left(\psi_{1}\right)=0.99(5)$ and $C\left(\boldsymbol{\psi}_{2}\right)=0.01(5)$, that is to say the refined structure is coplanar and the contribution from out-of-plane canting is zero within the error of these data. The final refined profile is presented in Figure 10 and the final magnetic structure in Figure 11.

\footnotetext{
${ }^{2}$ this criteria for this transformation is that the Coirreducible Representation (CIR) derived from the irreducible representation is real
} 


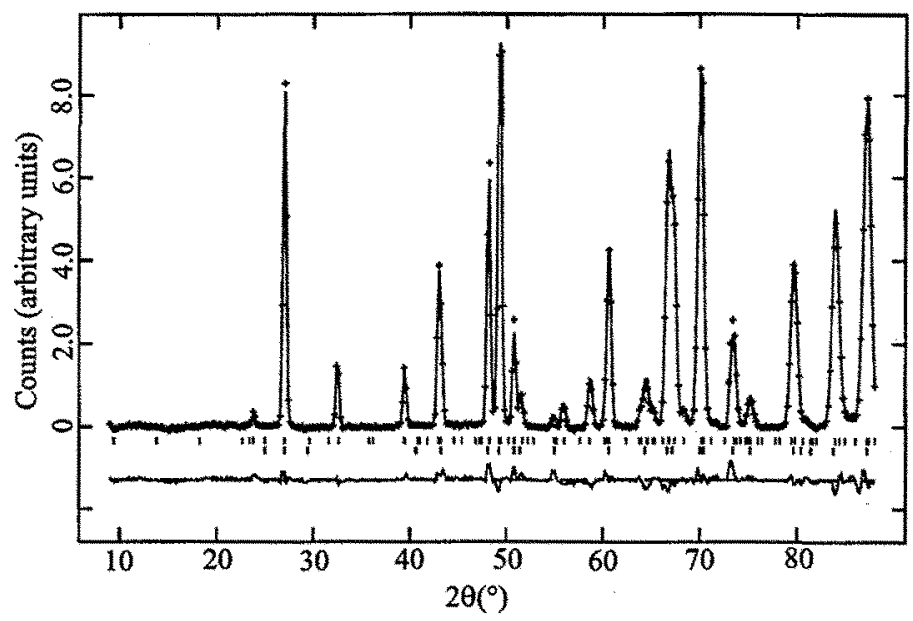

Figure 10: Experimental and calculated diffraction patterns for $\mathrm{AgFe}_{3}\left(\mathrm{SO}_{4}\right)_{2}(\mathrm{OD})_{6}$ at $1.5 \mathrm{~K}$. Magnetic and crystallographic reflections are indicated by the upper and lower tick marks respectively.

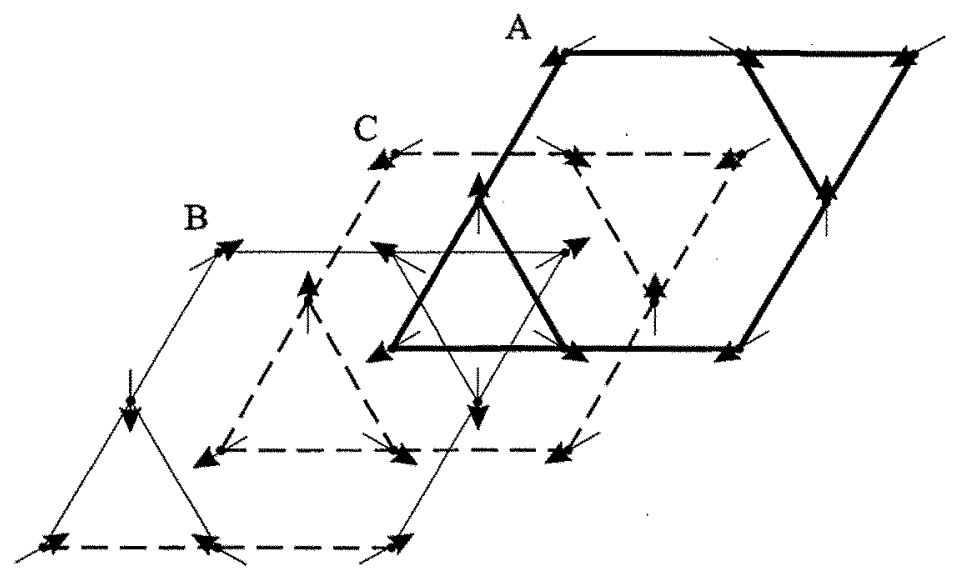

Figure 11: Magnetic structure of $\mathrm{AgFe}_{3}\left(\mathrm{SO}_{4}\right)_{2}(\mathrm{OD})_{6}$. 


\subsection{Discussion of the magnetic structure of $\mathrm{AgFe}_{3}\left(\mathrm{SO}_{4}\right)_{2}(\mathrm{OD})_{6}$}

The magnetic structure that is refined is a triangular structure, that is to say the neighbouring moments are related by $120^{\circ}$. This is what we would naively expect for a triangular array of antiferromagnetically coupled spins. The Group Theory arguments we have used indicate that only particular orientations are possible for this configuration. As we will show in the practicals that accompany this course, the out-of-plane component that is only allowed in $\Gamma_{2}$ is important at higher temperatures and leads to the formation of an umbrella structure.

\section{Summary of course}

As stated in the introduction, this course was intended to explain how to describe a magnetic structure in terms of a propagation vector and some of its associated basis vectors. The examples given show how the different possible types of magnetic structure lead directly from this description. The second part of this text has been devoted to magnetic symmetry analysisRepresentational Analysis. The Group Theory calculations that this involves are tedious, but now computer programs exist that perform these calculations in seconds. The basis vectors that result simplify greatly the processes of finding a magnetic structure, and can facilitate their correct description.

\section{Further reading}

Much inspiration, of varying levels, has been taken from a number of works on magnetic structures and magnetic symmetry analysis. For a first step into these subjects the References $[1,4,5,6,7]$ are particular suitable. Reference [7] presents a clear introduction into the technical aspects of magnetic neutron scattering.

\section{Acknowledgements}

ASW would like to thank the Marie-Curie project of the EC for support and the Grenoble school of magnetism for their tuition, encouragement, and patience.

\section{References}

[1] Yu.A. Izymov, V.E. Naish and R.P. Ozerov, 'Neutron Diffraction of Magnetic Materials', Consultants Bureau, New York, 1991.

[2] C.G. Shull and J.S. Smart, Phys. Rev. 76, 1256 (1949).

[3] D. Gignoux, J.C. Gomez Sal, J. Magn. Magn. Mat. 1, 203 (1976).

[4] J. Rossat-Mignod in: 'Methods in Experimental Physics', ed. K. Sköld and D.L. Price (Academic Press, 1987). J. Rossat-Mignod in 'Systematics and the Propeties of the Lanthanides', Ed. S.P. Sinha, D. Reidel Publishing (1983).

[5] P.J. Brown, Physica B 137, 31 (1986). 
[6] J. Schweizer, in: 'Hercules- Neutron and Synchrotron Radiation for Condensed Matter Research', Springer Verlag, Berlin (1994).

[7] F. Tasset, Recent developments in neutron scattering Proceedings of Les Houches École de Physique 2000.

[8] F. Tasset, Structures magnétiques et polarimétrie neutronique Proceedings of 'l'Ecole Magnétisme et Neutrons' de la Société Française de la Neutronique, a French Translation of Ref. [7] to appear in J. de Physique (2001).

[9] C.J. Bradley and A.P. Cracknell, 'The Mathematical Theory of Symmetry in Solids', Clarendon Press (Oxford, 1972).

[10] E.F. Bertaut, J. Appl. Phys, 33, 1138 (1962). E.F. Bertaut, Acta. Cryst. A24, 217 (1968) E.F. Bertaut, J. de Physique, Colloque C1, 462 (1971). E.F. Bertaut, J. Magn. Magn. Mat. 24, 267 (1981).

[11] Yu.A. Izyumov and V.E. Naish, J. Magn, Magn. Mat.12, 239 (1979); Yu.A. Izyumov and V.E. Naish, J. Magn, Magn. Mat.12, 249 (1979); Yu.A. Izyumov and V.E. Naish, J. Magn, Magn. Mat.13, 267 (1979).

[12] E.R. Hovestreydt, M.I. Aroyo and H. Wondratschek, J. Appl. Cryst. 25, 544 (1992).

[13] P. Czapnik and W. Sikora, unpublished work.

[14] J. Rodriguez-Carvajal, unpublished work.

[15] A.S. Wills, Physica B 276-278(2000), 680. Program available from $\mathrm{ftp}: / / \mathrm{ftp}$.ill.fr/pub/dif/sarah/

[16] A.S. Wills, A. Harrison, C. Ritter and R.I. Smith, Phys. Rev. B 61, 6156 (2000).

[17] A.S. Wills, Phys. Rev. B, 63, 064430 (2001).

[18] A.S. Wills, Proceedings of Highly Frustrated Magnetism 2000 Conference, to appear in Can. J. Phys. (2001).

[19] O.V. Kovalev, 'Irreducibe Representations of the Space Groups', Gordon and Breach (New York, 1961). 